\title{
Characterization of Subclinical Thyroid Dysfunction From Cardiovascular and Metabolic Viewpoints The Suita Study
}

\author{
Naoyuki Takashima, MD; Yasuharu Niwa, PhD; Toshifumi Mannami, MD*,**; \\ Hitonobu Tomoike, MD*; Naoharu Iwai, MD
}

\begin{abstract}
Background Subclinical hypothyroidism, defined as high serum thyroid-stimulating hormone (TSH) levels and normal serum free-triiodothyronine (fT3) and serum free-thyroxine (fT4) levels, is a common medical problem among the elderly, but it is unclear whether it should be treated with thyroid hormone replacement therapy. Methods and Results A cross-sectional study of 3,607 participants in a community health survey in Suita, in the northern part of Osaka, was performed. Participants were categorized into 5 groups: normal, hyperthyroidism, hypothyroidism, subclinical hypothyroidism, and subclinical hyperthyroidism. The association between each group and various phenotypes was examined, in relation to cardiovascular disease and metabolic syndromes. Serum TSH levels increased and fT3 and fT4 levels decreased with age. A total of $14.6 \%$ of subjects aged 70-80 years and $20.1 \%$ of subjects aged older than 80 years were classified as having subclinical hypothyroidism. Subclinical hypothyroidism was not associated with glycol-hemoglobin A1c, body mass index, pulse rate, hypertension, total cholesterol, high-density lipoprotein cholesterol or triglyceride levels or intima-media thickness. It was only associated with higher fasting blood glucose and glycol-hemoglobin A1c levels compared with euthyroidism. Conclusions The present observation does not support the need for treatment of subclinical hypothyroidism or subclinical hyperthyroidism. (Circ J 2007; 71: 191-195)
\end{abstract}

Key Words: Blood sugar; Diabetes; Lipids; Subclinical hyperthyroidism; Subclinical hypothyroidism

$\mathbf{S}$ ubclinical hypothyroidism ( $\mathrm{SCH})$, defined as high serum thyroid-stimulating hormone (TSH) levels and normal levels of serum free-triiodothyronine (fT3) and serum free-thyroxine (fT4), is a common medical problem among the elderly. The prevalence of $\mathrm{SCH}$ has been reported to be $4-10 \%$ in the general population and up to $20 \%$ in women older than 60 years ${ }^{1-3}$ The incidence of SCH is $2.1-3.8 \%$ per year in thyroid-antibody-positive subjects and $0.3 \%$ per year in thyroid-antibody-negative subjects.

Serum lipid levels in SCH have been reported as either normal $^{5}$ or elevated.,7 In the Troms $\varnothing$ study, low-density lipoprotein-cholesterol (LDL-C) levels were significantly higher in subjects with $\mathrm{SCH}$ compared with controls ${ }^{7}$ and, moreover, they were reduced with thyroxine treatment. In addition, associations between left ventricular function and SCH have been widely investigated, but the findings are controversial. Some studies have shown an association between $\mathrm{SCH}$ and poor left ventricular function and others have not? Moreover, the positive association between arterial stiffness and hypothyroidism, even in the subclinical stage, has been reported9,10 Subclinical hyperthyroidism has been associated with a higher prevalence of atrial fibrillation (AF) and increased heart rate 8 but not with elevated serum lipid levels6.

(Received August 17, 2006; revised manuscript received October 31, 2006; accepted November 20, 2006)

Departments of Epidemiology, *Preventive Cardiology, National Cardiovascular Center, Suita and **Hygiene and Public Health, Social Medicine, Faculty of Medicine, Kagawa University, Kagawa, Japan Mailing address: Naoyuki Takashima, MD, Department of Epidemiology, National Cardiovascular Center, 5-7-1 Fujishirodai, Suita 5658565, Japan. E-mail: ntaka@ri.ncvc.go.jp
In the present study we investigated whether subclinical thyroid dysfunction in Japanese individuals is associated with various phenotypes related to cardiovascular disease and metabolic syndromes.

\section{Methods}

\section{Study Population}

The selection criteria and design of the Suita study have been described previously!1-13 Serum TSH, fT3, and fT4 levels were measured in 3,607 subjects who were not being treated for thyroid disease. The present study was approved by the Ethics Committee of the National Cardiovascular Center, and all subjects provided written informed consent. We categorized patients into 5 groups: normal (normal levels of serum TSH $[0.436-3.78 \mu \mathrm{U} / \mathrm{ml}]$, fT3 [2.1-4.1 pg/ml] and fT4 [1.0-1.7 ng/dl]), hyperthyroidism (low levels of TSH and high levels of fT3 and/or fT4), hypothyroidism (high levels of TSH and low levels of fT3 and/or fT4), SCH (high levels of TSH and normal levels of fT3 and fT4), and subclinical hyperthyroidism (low levels of TSH and normal levels of fT3 and fT4) $!^{4}$ Body mass index (BMI) was calculated as body weight $(\mathrm{kg})$ divided by height in square meters.

The intima-media thickness (IMT) was measured on longitudinal scan of the common carotid artery at a point $10 \mathrm{~mm}$ proximal from the beginning of the dilation of the bulb.1

Serum TSH, fT3, and fT4 Levels

Fasting serum samples were collected at study entry and stored at $-80^{\circ} \mathrm{C}$ until tests were run. Serum TSH was mea- 

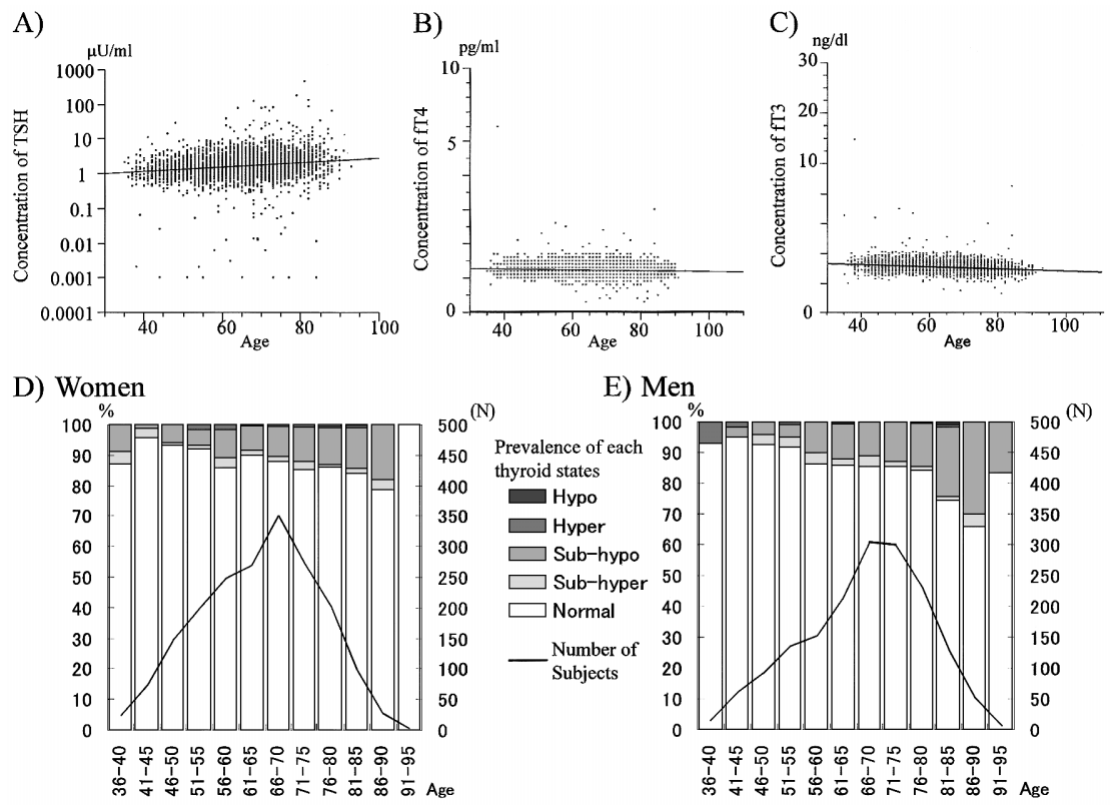

E) Men

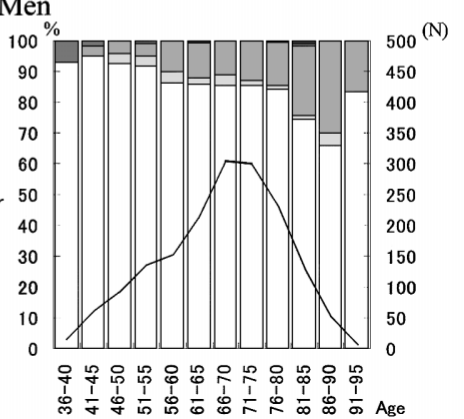

Fig 1. Serum TSH, fT3, and fT4 hormone levels and age. $(\mathrm{A}-\mathrm{C})$ Correlation between serum TSH (A), fT3 (B), and fT4 levels (C) and age are plotted (grey dot). Serum TSH levels increased with age. There was a linear correlation between log transferred serum TSH levels and age. Serum fT3 and fT4 levels decreased with age. There was a linear correlation between these thyroid hormone levels and age $(\mathrm{p}<0.01)$. (D-E) The prevalence of women (D) and men (E) in each thyroid state according to age is shown. The total number of subjects according to age is shown by the black line. The prevalence of subclinical hypothyroid in both men and women increased with age. However the prevalence of subclinical hyperthyroidism did not increase with age. TSH thyroid-stimulating hormone; fT3, serum free-triiodothyronine; fT4 serum free-thyroxine. sured by a chemiluminescent immunoassay kit (Mitsubishi Kagaku BCL, Abbott Laboratories, Chicago, IL, USA), as were serum fT3 and fT4 levels (Mitsubishi Kagaku BCL, Bayer, Leverkusen, Germany).

\section{Statistical Analysis}

Values are expressed as mean \pm standard deviation. All statistical analyses were performed with the JMP statistical package (SAS Institute Inc, Cary, NC, USA). One-way analysis of variance tests were used to determine whether an association existed among thyroid status and BMI (adjusted for age and sex), systolic and diastolic blood pressures (SBP: adjusted for age, BMI, and sex; DBP: adjusted for age, $\mathrm{BMI}$, and sex), pulse rate (PR; adjusted for age and sex), glycol-hemoglobin A1c (HbA1c: adjusted for age, BMI, and sex), fasting blood glucose levels (FBG: adjusted for age, BMI, and sex), high-density lipoprotein (HDL: adjusted for cholesterol BMI, sex, age, number of cigarettes/day, and alcohol consumption [g/day]), total cholesterol (TC: adjusted for age, BMI, and sex), triglycerides (TG: adjusted for age, BMI, and sex) and IMT. Simple correlation analyses were used to determine whether an association existed between normal states and each thyroid state, as well as between the variables assessed after adjusting for confounding factors. Logistic analysis was used to determine whether an association existed among thyroid status and the prevalence of AF.

\section{Results}

Serum concentrations of TSH increased and serum concentrations of fT3 and fT4 decreased with age (Figs 1A-C). Similarly, the number of men and women with $\mathrm{SCH}$ also increased with age (Figs 1D,E). Overall, 11.6\% of women aged 71-80 years and $13.8 \%$ of women aged older than 80 years were classified into the SCH category (Fig 1D). For men, $13.4 \%$ aged $71-80$ years and $24.5 \%$ older than 80 years were classified into the SCH category (Fig 1E).

The characteristics of the study population are shown in Table 1. Participants included 3,130 normal subjects, 19 with hyperthyroidism, 4 with hypothyroidism, 77 with sub- clinical hyperthyroidism, and 377 with SCH. The prevalence of SCH was $10.45 \%$ and that of subclinical hyperthyroidism was $2.13 \%$. Compared with normal individuals, subjects with $\mathrm{SCH}$ were significantly older $(69.02 \pm 10.43$ years vs $64.22 \pm 11.29$ years, respectively; $p<0.05)$ and had significantly lower serum fT3 levels $(2.99 \pm 0.33 \mathrm{ng} / \mathrm{dl}$ vs $3.12 \pm 0.32 \mathrm{ng} / \mathrm{dl}$, respectively; $\mathrm{p}<0.01$, adjusted for age and sex) and fT4 levels $(1.09 \pm 0.19 \mathrm{pg} / \mathrm{ml}$ vs $1.24 \pm 0.15 \mathrm{pg} / \mathrm{ml}$, respectively; $\mathrm{p}<0.01$, adjusted for age and sex). On the other hand, when individuals with subclinical hyperthyroidism were compared with normal subjects, they showed significantly higher concentrations of fT4 $(1.34 \pm 0.18 \mathrm{pg} / \mathrm{ml}$ vs $1.24 \pm 0.15 \mathrm{pg} / \mathrm{ml}$, respectively; $\mathrm{p}<0.01$, adjusted for age and sex). Subjects with overt hyperthyroidism had significantly lower HDL $(\mathrm{p}<0.01)$ and TC levels $(\mathrm{p}<0.01)$ and higher PR $(p<0.01)$ than subjects with normal thyroid function. Subjects with overt hypothyroidism had significantly higher TC levels $(\mathrm{p}<0.01)$ than subjects with normal thyroid function. Characteristics associated with hyper- and hypothyroid subjects were as reported previously 15

Characteristics of subjects with normal and subclinical thyroid dysfunction are shown in Table 2. The PR among subjects not being treated for arrhythmia was not significantly associated with subclinical hyperthyroidism or $\mathrm{SCH}$ after adjusting for age and sex. Blood pressure among subjects not being treated for hypertension was not significantly associated with subclinical hyperthyroidism or $\mathrm{SCH}$ after adjusting for appropriate confounding factors. Among subjects not being treated for hypertriglyceridemia, TG levels were not significantly associated with subclinical hyperthyroidism or SCH compared with normal subjects. HDL-cholesterol and TC levels were not significantly associated with subclinical hyperthyroidism or SCH subjects not being treated for hyperlipidemia. Among all subjects not receiving treatment for diabetes, FBG was significantly associated with SCH $(\mathrm{p}<0.01)$. Subjects with subclinical hyperthyroidism had significantly higher HbA1c $(\mathrm{p}<0.05)$ and FBG $(p<0.01)$ levels than normal subjects. The numbers of subjects not being treated for each disease is shown in Table 2.

The prevalence of AF in subjects with subclinical hyper- 
Table 1 Characteristics of the Study Population

\begin{tabular}{|c|c|c|c|c|c|c|}
\hline & $\begin{array}{l}\text { Normal } \\
\text { thyroid }\end{array}$ & Hyperthyroidism & Hypothyroidism & $\begin{array}{c}\text { Subclinical } \\
\text { hyperthyroidism }\end{array}$ & $\begin{array}{c}\text { Subclinical } \\
\text { hypothyroidism }\end{array}$ & Total \\
\hline$N(\%)$ & $3,130(86.78)$ & $19(0.53)$ & $4(0.11)$ & $77(2.13)$ & $377(10.45)$ & $3,607(100)$ \\
\hline Male (\%) & $1,442(46.07)$ & $5(26.32)$ & $3(75.0)$ & $39(50.65)$ & $203(53.85)$ & $1,692(44.55)$ \\
\hline Age (years) & $64.22 \pm 11.29$ & $62.16 \pm 12.85$ & $76.50 \pm 8.43$ & $63.94 \pm 10.85$ & $69.02 \pm 10.43^{\ddagger}$ & $64.70 \pm 11.29$ \\
\hline$B M I$ & $22.82 \pm 3.11$ & $22.73 \pm 3.48$ & $24.02 \pm 0.58$ & $22.77 \pm 3.03$ & $22.84 \pm 3.34$ & $22.82 \pm 3.14$ \\
\hline $\mathrm{SBP}(\mathrm{mmHg})$ & $129.24 \pm 19.65$ & $131.32 \pm 20.63$ & $135.50 \pm 10.47$ & $125.57 \pm 15.45$ & $131.93 \pm 19.06$ & $129.41 \pm 19.69$ \\
\hline$D B P(m m H g)$ & $77.82 \pm 10.09$ & $76.11 \pm 11.95$ & $71.50 \pm 15.37$ & $75.03 \pm 10.76$ & $77.97 \pm 9.61$ & $77.74 \pm 10.17$ \\
\hline$P R$ (beats/min $)^{\S}$ & $66.10 \pm 8.25$ & $72.42 \pm 9.74^{\ddagger}$ & $62.00 \pm 3.27$ & $67.12 \pm 9.38$ & $66.51 \pm 8.35$ & $66.20 \pm 8.38$ \\
\hline$F B G(m g / d l)^{\mathscr{I}}$ & $99.75 \pm 21.32$ & $105.16 \pm 40.89$ & $99.00 \pm 4.97$ & $106.79 \pm 38.83^{\dagger}$ & $98.64 \pm 18.68$ & $99.79 \pm 21.67$ \\
\hline$H b A l c(\%)^{\pi}$ & $5.49 \pm 0.74$ & $5.47 \pm 0.86$ & $5.57 \pm 0.31$ & $5.73 \pm 1.49^{\dagger}$ & $5.54 \pm 0.77$ & $5.50 \pm 0.77$ \\
\hline$H D L-C(m g / d l)$ & $60.56 \pm 15.52$ & $51.11 \pm 15.81^{\ddagger}$ & $55.25 \pm 16.80$ & $58.71 \pm 17.01$ & $58.47 \pm 16.01$ & $60.20 \pm 15.61$ \\
\hline$T C(m g / d l)$ & $208.26 \pm 32.09$ & $185.58 \pm 35.83^{\ddagger}$ & $247.25 \pm 55.05^{\dagger}$ & $206.79 \pm 33.26$ & $206.34 \pm 35.48$ & $207.99 \pm 32.53$ \\
\hline$T G(m g / d l)$ & $106.90 \pm 74.77$ & $111.74 \pm 64.30$ & $156.00 \pm 61.36$ & $123.12 \pm 72.03$ & $113.30 \pm 69.72$ & $108.16 \pm 74.59$ \\
\hline Prevalence of MI (\%) & $40(1.28)$ & $0(0.00)$ & $0(0.00)$ & $2(2.60)$ & $7(1.86)$ & $49(1.36)$ \\
\hline Prevalence of CVA (\%) & $89(2.84)$ & $0(0.00)$ & $0(0.00)$ & $3(3.90)$ & $13(3.45)$ & $105(2.91)$ \\
\hline Prevalence of $A F(\%)$ & $44(1.41)$ & $0(0.00)$ & $0(0.00)$ & $2(2.60)$ & $15(3.98)$ & $61(1.69)$ \\
\hline$T S H(\mu U / m l)$ & $1.723 \pm 0.824$ & $0.10 \pm 0.20$ & $209.85 \pm 171.57+$ & $0.27 \pm 0.13 *$ & $8.32 \pm 11.42^{\ddagger}$ & $2.60 \pm 9.51$ \\
\hline$f T 4(n g / d l)$ & $1.24 \pm 0.15$ & $2.26 \pm 0.97^{\ddagger}$ & $0.35 \pm 0.0^{\ddagger}$ & $1.34 \pm 0.18^{\frac{5}{ \pm}}$ & $1.09 \pm 0.19^{\ddagger}$ & $1.23 \pm 0.20$ \\
\hline$f T 3(\mathrm{pg} / \mathrm{ml})$ & $3.12 \pm 0.32$ & $6.75 \pm 5.77^{\ddagger}$ & $1.65 \pm 0.29+$ & $3.18 \pm 0.46$ & $2.99 \pm 0.33^{\frac{t}{t}}$ & $3.12 \pm 0.59$ \\
\hline
\end{tabular}

${ }^{\dagger} p<0.05$ and $\stackrel{\ddagger}{\ddagger}<0.01$.

$P$ values are for comparisons with normal thyroid subjects after adjustment for appropriate confounding factors.

Values are mean \pm standard deviation.

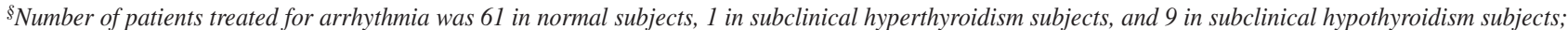
"number of patients treated for diabetes was 150 in normal subjects, 1 in hyperthyroidism subjects, 7 in subclinical hyperthyroidism subjects, and 22 in subclinical hypothyroidism subjects.

BMI, body mass index; SBP, systolic blood pressure; DBP, diastolic blood pressure; PR, pulse rate; FBG, fasting blood glucose levels; Hb, hemoglobin; HDL, high-density lipoprotein; C, cholesterol; TC, total cholesterol; TG, triglyceride; MI, myocardial infraction; CVA, cerebrovascular accident; AF, atrial fibrillation; TSH, thyroid-stimulating hormone; fT4, free-thyroxine; fT3, free-triiodothyronine.

Table 2 Characteristics of the Subjects in the Normal and Subclinical Thyroidism Groups

\begin{tabular}{|c|c|c|c|c|}
\hline & Normal thyroid & $\begin{array}{c}\text { Subclinical } \\
\text { hyperthyroidism }\end{array}$ & $\begin{array}{c}\text { Subclinical } \\
\text { hypothyroidism }\end{array}$ & $\begin{array}{l}\text { ANOVA } \\
\text { p-value** }\end{array}$ \\
\hline$N$ & 3,130 & 77 & 377 & \\
\hline$B M I$ & $22.82 \pm 3.11$ & $22.77 \pm 3.03$ & $22.84 \pm 3.34$ & $N S$ \\
\hline$N^{\dot{⿱}}$ & 2,355 & 57 & 270 & \\
\hline $\mathrm{SBP}(\mathrm{mmHg})$ & $124.95 \pm 18.37$ & $122.53 \pm 20.00$ & $127.74 \pm 17.98$ & $N S$ \\
\hline$D B P(m m H g)$ & $76.67 \pm 9.98$ & $73.49 \pm 10.99$ & $76.93 \pm 9.50$ & $N S$ \\
\hline$N^{\S}$ & 3,057 & 75 & 364 & \\
\hline$P R$ (beats $/ \mathrm{min})$ & $66.12 \pm 8.23$ & $67.33 \pm 9.40$ & $66.47 \pm 8.27$ & $N S$ \\
\hline$N^{q /}$ & 2,976 & 70 & 355 & \\
\hline $\mathrm{HbAlc}$ & $5.40 \pm 0.56$ & $5.56 \pm 1.34 *$ & $5.41 \pm 0.48$ & $<0.05$ \\
\hline$F B G(m g / d l)$ & $97.07 \pm 15.27$ & $103.6 \pm 36.73^{\dagger}$ & $95.58 \pm 11.57^{\dagger}$ & $<0.0001$ \\
\hline$N^{i \dagger}$ & 3,084 & 73 & 371 & \\
\hline$T G(m g / d l)$ & $105.95 \pm 74.12$ & $121.52 \pm 75.98$ & $112.73 \pm 69.70$ & $N S$ \\
\hline$N^{\|}$ & 2,726 & 63 & 320 & \\
\hline$H D L(m g / d l)$ & $60.58 \pm 15.65$ & $58.40 \pm 17.36$ & $58.37 \pm 16.41$ & $N S$ \\
\hline$T C(m g / d l)$ & $207.78 \pm 32.47$ & $207.40 \pm 34.81$ & $205.44 \pm 36.39$ & $N S$ \\
\hline
\end{tabular}

$*<0.05$ and $^{\dagger}<0.01$.

**P values are for comparisons with normal thyroid subjects after adjustment for appropriate confounding factors.

Values are mean \pm standard deviation.

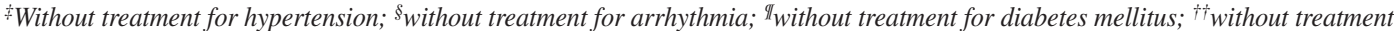
for hypertriglyceridemia; "without treatment for hyperlipidemia. ANOVA, analysis of variance. Other abbreviations see in Table 1.

thyroidism was higher than that in normal subjects $(3.98 \%$ vs $1.41 \%$ ) but the difference was not statistically significant. Subjects who had been diagnosed with AF were included with those with $\mathrm{AF}$.

A total of $12 \%$ of subjects with SCH had high serum TSH levels $(>10 \mu \mathrm{U} / \mathrm{ml})$. Subjects with $\mathrm{SCH}$ and TSH levels $>10 \mu \mathrm{U} / \mathrm{ml}$ were older than subjects with $\mathrm{SCH}$ and TSH levels $=10 \mu \mathrm{U} / \mathrm{ml}(67.28 \pm 9.62$ years vs $71.07 \pm 9.70$ years, $\mathrm{p}<0.05)$. Subjects with $\mathrm{SCH}$ and high TSH levels $(>10 \mu \mathrm{U} / \mathrm{ml})$ did not have lower FBG, HbA1c or lipid levels compared with normal subjects (unpubl. data).
The correlation between laboratory data and fT3, fT4, and TSH levels in normal subjects is shown in Table 3. Serum fT4 and fT3 levels in normal subjects were significantly associated with various laboratory data after adjusting for appropriate confounding factors.

We examined whether an association between IMT and thyroid dysfunction existed (Table4). There were weak associations between thyroid states and IMT-mean, and IMT-max, but these disappeared after adjusting for age and sex. Multiple logistic analysis did not detect a significant correlation between thyroid state (normal or subclinical 
Table 3 Correlation Between Laboratory Data and Thyroid Hormone Level in Normal Subjects

\begin{tabular}{|c|c|c|c|c|c|c|c|c|c|}
\hline Thyroid state & $T G^{\dagger \dagger}$ & $F B G^{\mathscr{I}}$ & $H b A 1 c^{\mathbb{I}}$ & $T C^{\|}$ & $H D L^{\|}$ & $B M I$ & $P R^{\S}$ & $S B P^{\ddagger}$ & $D B P^{\ddagger}$ \\
\hline $\begin{array}{l}\text { No. of subjects } \\
\text { TSH }\end{array}$ & 3,084 & 2,976 & 2,976 & 2,726 & 2,726 & 3,130 & 3,057 & 2,355 & 2,355 \\
\hline$P$ & 0.0015 & $N S$ & $N S$ & 0.015 & 0.0055 & $N S$ & $N S$ & $N S$ & $N S$ \\
\hline$*$ & 5.21 & 0.20 & 0.0090 & 1.83 & -0.92 & 0.045 & -0.10 & -0.24 & 0.12 \\
\hline \multicolumn{10}{|l|}{$\begin{array}{l}\text { Normal thyroid } \\
\quad f T 3\end{array}$} \\
\hline$P$ & 0.0043 & $N S$ & $N S$ & $N S$ & $<0.0001$ & $<0.0001$ & $N S$ & $N S$ & $N S$ \\
\hline * & 12.16 & 0.15 & -0.023 & 0.22 & -4.01 & 1.06 & 0.12 & 1.75 & 0.64 \\
\hline \multicolumn{10}{|l|}{$f T 4$} \\
\hline$P$ & 0.039 & 0.0006 & 0.030 & 0.0010 & 0.071 & $N S$ & $<0.0001$ & 0.002 & 0.023 \\
\hline$*$ & 18.00 & 6.03 & 0.14 & 13.09 & 3.16 & 0.43 & 4.83 & 7.06 & 2.95 \\
\hline
\end{tabular}

* Correlation coefficient with each value.

$P$ values are coefficient of correlation; $p$ values are for comparisons with normal thyroid subjects after adjustment for appropriate confounding factors.

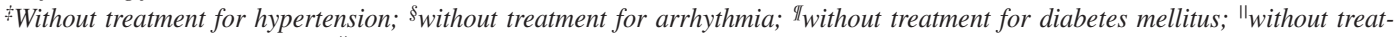
ment for hypertriglyceridemia; "without treatment for hyperlipidemia.

Abbreviations see in Table 1.

Table 4 Correlation Between Thyroid Status and IMT

\begin{tabular}{|c|c|c|c|c|c|c|}
\hline & $\begin{array}{l}\text { Normal } \\
\text { thyroid }\end{array}$ & Hyperthyroidism & Hypothyroidism & $\begin{array}{c}\text { Subclinical } \\
\text { hyperthyroidism }\end{array}$ & $\begin{array}{c}\text { Subclinical } \\
\text { hypothyroidism }\end{array}$ & $\begin{array}{l}\text { ANOVA } \\
p \text { value }\end{array}$ \\
\hline$N$ & 2,819 & 17 & 4 & 65 & 321 & \\
\hline$I M T-\max (\mathrm{mm})$ & $1.30 \pm 0.55$ & $1.21 \pm 0.63$ & $1.73 \pm 0.97$ & $1.25 \pm 0.53$ & $1.40 \pm 0.58$ & 0.048 \\
\hline Residual IMT-max* & $0.002 \pm 0.491$ & $-0.015 \pm 0.492$ & $0.089 \pm 0.892$ & $-0.052 \pm 0.467$ & $-0.009 \pm 0.520$ & 0.892 \\
\hline Residual IMT-max ${ }^{\dagger}$ & $0.001 \pm 0.784$ & $-0.046 \pm 0.506$ & $0.094 \pm 0.855$ & $-0.064 \pm 0.463$ & $-0.001 \pm 0.510$ & 0.786 \\
\hline$N$ & 2,818 & 17 & 4 & 65 & 320 & \\
\hline IMT-mean (mm) & $0.821 \pm 0.13$ & $0.79 \pm 0.13$ & $0.93 \pm 0.09$ & $0.83 \pm 0.13$ & $0.83 \pm 0.13$ & 0.042 \\
\hline Residual IMT-mean* & $0.0004 \pm 0.105$ & $-0.004 \pm 0.108$ & $0.022 \pm 0.496$ & $0.018 \pm 0.118$ & $-0.008 \pm 0.112$ & 0.581 \\
\hline Residual IMT-mean ${ }^{\dagger}$ & $0.000 \pm 0.103$ & $-0.301 \pm 0.103$ & $0.014 \pm 0.035$ & $0.014 \pm 0.111$ & $-0.005 \pm 0.110$ & 0.821 \\
\hline
\end{tabular}

Values are mean \pm standard deviation.

*Aadjusted for age and sex; ‘ adjusted by age, BMI, SBP, FBS, number of cigarettes/day, TG, TC and HDL.

IMT, intima-media thickness. Other abbreviations see in Tables 1,2.

thyroid dysfunction) and the prevalence of atherosclerotic vascular diseases, such as cerebral infarction, transient cerebral ischemic attack, cerebral stroke, acute myocardial infarction and angina pectoris ( $\mathrm{p}=0.090$, unpubl. data).

\section{Discussion}

In this large cross-sectional study of subclinical thyroid dysfunction in Japanese subjects, although the prevalence of SCH increased with age overall, the prevalence of $\mathrm{SCH}$ in elderly patients was lower than that reported in other studies, particularly in women: $18.8 \%$ for men older than 75 years and $12.7 \%$ for women older than 75 years. This finding differs from that of previous studies in which the prevalence of $\mathrm{SCH}$ was higher in elderly women than in elderly men1,3,6 In the Colorado study, the prevalence of $\mathrm{SCH}$ was $16 \%$ in men older than 75 years and $21 \%$ in women older than 75 years! The differences in these trends might be due to different genetic, ethnic or environmental backgrounds of the subjects. On the other hand, the prevalence of subclinical hyperthyroidism was $2.13 \%$ in our study, which is similar to that in the Colorado study $(2.1 \%)$.

We found that the presence of $\mathrm{SCH}$ was associated with lower FBG levels and that subclinical hyperthyroidism was associated with higher FBG and HbAlc levels. Moreover, our results also indicate that thyroid hormone levels in normal subjects are significantly associated with various laboratory data, including FBG and $\mathrm{HbA1c}$ levels. Compared with normal subjects, serum thyroid hormone levels in subjects with $\mathrm{SCH}$ were lower and levels in subjects with subclinical hyperthyroidism were higher. Therefore, lower or higher levels of thyroid hormone (within the normal range) in subclinical thyroid dysfunction might influence glucose metabolism.

We did not observe any significant association between subclinical thyroid dysfunction and lipid metabolism, which was consistent with a previous US study5 However, other studies in Norway and Australia have reported dyslipidemia in subjects with $\mathrm{SCH}^{6,7}$ We examined whether higher TSH levels $(>10 \mu \mathrm{U} / \mathrm{ml})$ in subjects with $\mathrm{SCH}$ were associated with lipid metabolism, but did not find any significant association between lipid metabolism and TSH levels $>10 \mu \mathrm{U} / \mathrm{ml}$ in subjects with $\mathrm{SCH}$ compared with normal subjects. Moreover, higher TSH levels did not show any association with FBG levels. These results might be related to aging, because hypofunction of the endocrine glands occurs with age. Serum lipid, FBG, and HbA1c levels were sustained in subjects with high TSH levels and $\mathrm{SCH}$.

We did not observe any significant association between subclinical thyroid dysfunction and IMT, which suggests that subclinical thyroid dysfunction might not be related to an increased risk of atherosclerosis. Moreover, we did not find any significant association between $\mathrm{SCH}$ and previous history of arteriosclerotic vascular diseases. However, this result was not consistent with previous studies, 8,10 possibly because of the size of our study population and different 
surrogate markers for atherosclerosis.

The necessity for thyroid hormone replacements therapy for SCH is not supported by the present study results. The signs and symptoms of hypothyroidism are bradycardia, mild hypertension and hyperlipidemia, which might accelerate atherosclerosis in subjects with hypothyroidism 16 We examined the association between $\mathrm{SCH}$ and these symptoms or signs and did not observe an association between $\mathrm{SCH}$ and blood pressure or serum lipid levels. The association between lipid profile and $\mathrm{SCH}$ has been previously reported in Norway and Australia ${ }^{6,7}$ and the different results might relate to differences in genetic background, life style and BMI. In the Troms $\varnothing$ study? the subjects were obese and those with $\mathrm{SCH}$ were even more obese than normal subjects. The difference in BMI between those and our investigations might induce different patterns of lipid metabolism. We did not observe any significant association between $\mathrm{SCH}$ and the symptoms or signs associated with hypothyroidism. Moreover, we did not find an association between $\mathrm{SCH}$ and IMT, as a surrogate marker for atherosclerosis, and did not find an association between SCN and past history of atherosclerotic disease. These results do not support the need for treatment of SCH in Japanese subjects. However, our investigation was a cross-sectional study, so the duration of SCH was not considered in the analysis. Our results do not completely deny that subjects with long-term $\mathrm{SCH}$ have increased risk of atherosclerosis.

In conclusion, we examined the association between subclinical thyroid dysfunction and various factors in a general population. We only found an association between glucose levels and subclinical thyroid dysfunction. The differences in serum glucose levels among the thyroid states $(\mathrm{SCH}$, subclinical hyperthyroidism, and normal thyroid) were too small to lead to a recommendation for treatment of subclinical thyroid dysfunction and thus do not indicate a need for treatment of subclinical thyroid dysfunction in Japanese subjects.

\section{Acknowledgements}

This study was supported by a grant from the Program for Promotion of Fundamental Studies in Health Science of the National Institute of Biomedical Innovation. We acknowledge the contribution of the members of this study.

\section{References}

1. Canaris GJ, Manowitz NR, Mayor G, Ridgway EC. The Colorado thyroid disease prevalence study. Arch Intern Med 2000; 160: 526534.

2. Gharib H, Tuttle RM, Baskin HJ, Fish LH, Singer PA, McDermott MT. Subclinical thyroid dysfunction: A joint statement on management from the American Association of Clinical Endocrinologists, the American Thyroid Association, and the Endocrine Society. J Clin Endocrinol Metab 2005; 90: 581-585; discussion 586-587.

3. Sawin CT, Castelli WP, Hershman JM, McNamara P, Bacharach P. The aging thyroid: Thyroid deficiency in the Framingham Study. Arch Intern Med 1985; 145: 1386-1388.

4. Samuels MH. Subclinical thyroid disease in the elderly. Thyroid 1998; 8: 803-813.

5. Hueston WJ, Pearson WS. Subclinical hypothyroidism and the risk of hypercholesterolemia. Ann Fam Med 2004; 2: 351 - 355.

6. Walsh JP, Bremner AP, Bulsara MK, O'Leary P, Leedman PJ, Feddema P, et al. Thyroid dysfunction and serum lipids: A community-based study. Clin Endocrinol (Oxf) 2005; 63: 670-675.

7. Iqbal A, Jorde R, Figenschau Y. Serum lipid levels in relation to serum thyroid stimulating hormone and the effect of thyroxine treatment on serum lipid levels 7 in subjects with subclinical hypothyroidism: The Tromso Study. J Intern Med 2006; 260: 53-61.

8. Biondi B, Palmieri EA, Lombardi G, Fazio S. Effects of subclinical thyroid dysfunction on the heart. Ann Intern Med 2002; 137: $904-$ 914.

9. Dagre AG, Lekakis JP, Papaioannou TG, Papamichael CM, Koutras DA, Stamatelopoulos SF, et al. Arterial stiffness is increased in subjects with hypothyroidism. Int J Cardiol 2005; 103: 1-6.

10. Biondi B, Klein I. Hypothyroidism as a risk factor for cardiovascular disease. Endocrine 2004; 24: 1-13.

11. Mannami T, Konishi M, Baba S, Nishi N, Terao A. Prevalence of asymptomatic carotid atherosclerotic lesions detected by high-resolution ultrasonography and its relation to cardiovascular risk factors in the general population of a Japanese city: The Suita study. Stroke 1997; 28: 518-525.

12. Iwai N, Katsuya T, Mannami T, Higaki J, Ogihara T, Kokame K, et al. Association between SAH, an acyl-CoA synthetase gene, and hypertriglyceridemia, obesity, and hypertension. Circulation 2002; 105: $41-47$.

13. Kokubo Y, Iwai N, Tago N, Inamoto N, Okayama A, Yamawaki H, et al. Association analysis between hypertension and CYBA, CLCNKB, and KCNMB1 functional polymorphisms in the Japanese population: The Suita Study. Circ J 2005; 69: 138-142.

14. Surks MI, Ortiz E, Daniels GH, Sawin CT, Col NF, Cobin RH, et al. Subclinical thyroid disease: Scientific review and guidelines for diagnosis and management. JAMA 2004; 291: 228-238.

15. Dillmann WH, The thyroid. In: Bennett JC, editor. Cecil textbook of medicine, 20th edn. Philadelphia: WB Saunders; 1996; 1227-1245.

16. Irwin K, Kaie O. Thyroid hormone and the cardiovascular system. $N$ Engl J Med 2001; 344: 501 -509. 